\title{
Clinical and angiographic features of patients with coronary artery ectasia compared with stenotic coronary artery disease
}

\begin{abstract}
Background: Coronary artery ectasia (CAE) is frequently observed in patients undergoing coronary angiogrphy for evaluation of coronary artery disease (CAD). It is not clear whether $\mathrm{CAE}$ is a variant of $\mathrm{CAD}$ or a separate disease entity.

Objective: To compare the clinical and angiographic profile of patients having coronary artery ectasia (CAE) with those having stenotic coronary artery disease (CAD).

Materials And Methods: We studied 1176 consecutive patients who had coronary angiography for various clinical indications. Of these 297 patients were excluded because of incomplete data. The remaining 879 were divided into two groups Coronary Artery Ectasia Group (CAE Group) was found in 207 patients. These were compared with 672 patients (Stenotic CAD Group) who had coronary angiography for the same clinical indication during the same period. Stenotic CAD Group had no ectasia. CAE was defined as a vessel diameter of $\geq 1.5$ times that of nearest normal segment. A significant stenosis was defined as a $\geq 50 \%$ luminal loss of the diameter.
\end{abstract}

Results: Patients with CAE were 2years younger (56.4 \pm 9.9 years) than Stenotic CAD (58.6 \pm 9.5 years), $(\mathrm{p}<0.171)$. Males and females were in similar proportions in the two Groups. Diabetes was less frequent in CAE Group 76(36.7\%) as compared to Stenotic CAD Group 325(48.3\%) $(\mathrm{p}<0.003)$. Hypertension was present in similar proportions in both groups $117(56.5 \%)$ vs. $388(57.7 \%)(\mathrm{p}<0.774)$. More Arab patients were found in CAE Group 107(51.7\%) and more Indians in Stenotic CAD Group 384(57.1\%) $(\mathrm{p}<0.005)$

Coronary ectasia was most frequent in RCA $126(60.7 \%)$, followed by LAD in 82 patients $(39.6 \%)$ and least in LCx 73 patients $(35.3 \%)$. Conversely in stenotic CAD Group the LAD was more frequently affected $477(70.9 \%)$ as compared to CAE Group $117(56.5 \%)$ $(\mathrm{p}<0.0001)$. LCx had stenosis of $342(50.8 \%)$ in Stenotic CAD Group vs. $54(26.1 \%)$ in CAE Group ( $<0.0001$ ). RCA stenosis was observed in 507(75.3\%) in Stenotic CAD Group vs. 94(45.4\%) in CAE Group $(\mathrm{p}<0.0001)$.

Left main stem ectasia was observed in $15(7.3 \%)$ patients. LAD ectasia was found in $82(39.6 \%)$ patients. LCx ectasia was found in $73(35.3 \%)$ patients. RCA ectasia was observed in $126(60.7 \%)$ patients. In the CAE Group, only $32(15.5 \%)$ patients had isolated CAE without stenosis in any coronary artery. The majority $175(84.5 \%)$ patients had CAE in same vessels and stenosis in the same or other vessels.

Conclusion: Compared to $\mathrm{CAD}$, coronary ectasia occurred in younger male patients and inversely related to diabetes. ACS is the most common presentation for patients with CAE which suggest that it is not just a benign condition. The distribution of CAE among the three coronary arteries is also different to that in $\mathrm{CAD}$

Keywords: coronary artery disease, coronary artery ectasia, atherosclerosis, diabetes mellitus, acute myocardial infarction
Volume 9 Issue 4 - 2017

\author{
Abdul Rehman Abid,' Salem Abu Jalala, ${ }^{2}$ \\ Mohammad Gomaa Gad, ${ }^{3}$ AA Gehani ${ }^{4}$ \\ 'Associate Consultant Cardiology, The Heart Hospital, Hamad \\ Medical Corporation, Qatar \\ ${ }^{2}$ Senior Consultant Ccs-Adult Cardiology, The Heart Hospital, \\ Hamad Medical Corporation, Qatar \\ ${ }^{3}$ Fellow Adult Cardiology, The Heart Hospital, Hamad Medical \\ Corporation, Qatar \\ ${ }^{4}$ Senior Consultant Interventional Cardiologist, Director \\ Primary Coronary Angioplasty Program Chairman, The Heart \\ Center, Al Ahli Specialist Hospital, Qatar
}

\section{Correspondence: A A Gehani, Senior Consultant Interventional Cardiologist, Director, Primary Coronary Angioplasty Program, Chairman, The Heart Center, Al Ahli Specialist Hospital, Doha, Qatar, Tel 974-66004802, Email aa.gehani@gmail.com}

Received: July 07, 2017 | Published: July 25, 2017

\section{Introduction}

Coronary artery disease (CAD) is a major cause of morbidity and mortality worldwide. Each year, an estimated 635,000 Americans have a new coronary attack (defined as first hospitalized myocardia infarction (MI) or coronary heart disease related death) and 280,000 have a recurrent attack. ${ }^{1}$ Coronary artery ectasia (CAE) is a relatively common entity but its prevalence and relevance are not as well studied in the Middle Eastern region. In other parts of the World its prevalence varies from 1.5 to $5 \%$ in most literature; however, it was reported in as high as $10 \%$ in some nations. ${ }^{2}$ It is generally defined as segmental or diffuse dilatation of the coronary artery to more than 1.5 times the diameter of the adjacent segment of the same artery. ${ }^{2}$
It frequently coexists with aneurysms elsewhere, mostly involving the aorta.3,4 Its relationship to cardiovascular risk factors is variable., ${ }^{3,4}$ It occurs predominantly in males and is associated with acute coronary syndrome in one third of cases. ${ }^{5}$

The exact mechanism for the development of CAE is unknown, but evidence suggests a combination of genetic predisposition as well as the common risk factors known for CAD and abnormal vessel wall metabolism. ${ }^{6,7}$ Moreover elevated C-reactive protein levels may suggest an inflammatory process. ${ }^{6,7}$ It is estimated that $50 \%$ of CAE is related to atherosclerosis. ${ }^{8} \mathrm{CAE}$ often coexists with stenosis of coronary arteries. ${ }^{9}$ 
Coronary angiography remains the gold standard for the assessment of coronary artery anatomy including ectasia. Intravascular ultrasound is an excellent tool to assess luminal size and characterize arterial wall changes and Multi detector computed coronary angiography allows non-invasive diagnosis of CAE.$^{10}$ This study was designed to compare the clinical and angiographic profile of patients having coronary artery ectasia and those with stenotic coronary artery disease, but no ectasia in a Middle Eastern country Qatar.

\section{Patients and methods}

We studied 1176 consecutive patients. Of these 297 patients were excluded because of incomplete data. The remaining 879 were divided into two groups, Coronary Artery Ectasia Group (CAE Group) was found in 207 patients. These were compared with 672 patients (Stenotic CAD Group) who had coronary angiography for the same clinical indications during the same period. Stenotic CAD Group had stenosis $>50 \%$, without ectasia.

A Proforma was used for data collection. It included history, along with information regarding risk factors of $\mathrm{CAD}$, indication for coronary angiography and angiographic findings of each patient.

CAE was defined as a vessel diameter of $\geq 1.5$ times that of nearest normal segment. A significant stenosis was defined as a $\geq 50 \%$ loss of the diameter. In order to study the relation between age and the two pathologies, the study population was divided in to four age groups, $<40$ years, 41-50 years, 51-60years and >60years.

Patients with entirely normal coronaries or $<50 \%$ luminal narrowing were excluded unless they had ectasia.

The Primary end point was to compare the distribution of CAD as well as the coexistence of ectasia and stenotic CAD. Secondary clinical end points were to compare the cardiovascular risk factors in the two groups.

\section{Statistical analysis}

Statistical analysis was performed using the SPSS (release 17.0; SPSS, Inc; Chicago, IL) system for Windows. Continuous variables were expressed as mean $\pm \mathrm{SD}$ (Standard Deviation) while categorical variables were expressed as frequencies and percentages. Risk factors and severity of coronary artery disease in patients with and without coronary artery ectasia were compared in CAE Group and Stenotic CAD Group by applying Chi square test and $p$ values were calculated. A $p$ value of less than 0.05 was taken as significant.

\section{Results}

There were $812(92.4 \%)$ males and $67(7.6 \%)$ females in the entire study population. Males were $195(94.2 \%)$ in CAE Group and $617(91.8 \%)$ in Stenotic CAD Group. The mean age of study population was $58 \pm 9$.6years, however patients with CAE were younger (56.4 \pm 9.9 years) than Stenotic CAD (58.6 \pm 9.5 years), $(p<0.171)$. When divided into age groups, it was observed that majority of study patients $372(42.5 \%)$ were in the age group 51-60years which was similar in the CAE and Stenotic CAD groups. However there were significantly less older patients ( $>60$ years) in CAE group (29.5\%), as compared to $(40 \%)$, in Stenotic CAD Group $(\mathrm{p}<0.0001)$. Conversely there were significantly more young patients (<40years) in CAE group, $(7.7 \%)$, as compared to $(2.2 \%)$ in Stenotic CAD Group $(\mathrm{p}<0.0001)$. Table 1 shows the ethnic origin in the study patients. There were more Arab patients in CAE Group 107(51.7\%) vs 53(37.5\%) in the Stenotic CAD Group $(p<0.005)$. An inverse trend was observed from the Indian subcontinent with more patients in Stenotic CAD Group 384(57.1\%) vs. $89(43 \%)$ in CAE Group $(\mathrm{p}<0.005)$. Diabetes was less frequent in CAE Group 76(36.7\%) than in Stenotic CAD Group 325(48.3\%) $(\mathrm{p}<0.003)$, while hypertension was present in similar proportions in both groups $117(56.5 \%)$ vs. $388(57.7 \%)(\mathrm{p}<0.774)$. Combined Diabetes and hypertension was present in $306(34.8 \%)$ patients in the whole study population, however this combination was less frequent in CAE group 58(28\%) vs. 248(36.8\%) p $<0.20$.

Table I Baseline characteristics of the study population

\begin{tabular}{|c|c|c|c|}
\hline Characteristics & $\begin{array}{l}\text { CAE Group } \\
\mathrm{N}=207\end{array}$ & $\begin{array}{l}\text { Stenotic Cad } \\
\text { Group } N=672\end{array}$ & p value \\
\hline Age mean years & $56.4 \pm 9.9$ & $58.6 \pm 9.5$ & $<0 .|7|$ \\
\hline \multicolumn{4}{|l|}{ Age Groups } \\
\hline$\leq 40$ Years & $16(7.7 \%)$ & $15(2.2 \%)$ & $<0.0001$ \\
\hline $4 I-50$ Years & $35(16.9 \%)$ & $110(16.3 \%)$ & \\
\hline $51-60$ Years & $95(45.9 \%)$ & $279(41.5 \%)$ & \\
\hline$>60$ Years & $6 \mathrm{I}(29.5 \%)$ & $269(40 \%)$ & \\
\hline \multicolumn{4}{|l|}{ Gender } \\
\hline Male & 195(94.2\%) & $617(91.8 \%)$ & $<0.258$ \\
\hline Female & $12(5.8 \%)$ & $55(8.2 \%)$ & \\
\hline Diabetes mellitus & $76(36.7 \%)$ & $325(48.3 \%)$ & $<0.003$ \\
\hline Hypertension & II $7(56.5 \%)$ & $388(57.7 \%)$ & $<0.774$ \\
\hline DM and HTN & $58(28 \%)$ & $248(36.8 \%)$ & $<0.020$ \\
\hline Smoking & $110(53.1 \%)$ & $297(44.1 \%)$ & $<0.023$ \\
\hline Dyslipidemia & $61(29.5 \%)$ & $159(23.6 \%)$ & $<0.09$ \\
\hline Family history of CAD & $7(3.4 \%)$ & $17(2.5 \%)$ & $<0.509$ \\
\hline \multicolumn{4}{|l|}{ Ethnic Origin } \\
\hline Arabs & $107(5 \mid .7 \%)$ & $253(37.6 \%)$ & $<0.005$ \\
\hline Indian Subcontinent & $89(43 \%)$ & $384(57.1 \%)$ & \\
\hline Far East & $5(2.4 \%)$ & $21(3.1 \%)$ & \\
\hline Caucasians & $6(2.9 \%)$ & $14(2.1 \%)$ & \\
\hline
\end{tabular}

CAD: Coronary Artery Disease; DM: Diabetes Mellitus; HTN: Hypertension

Non ST elevation Acute Coronary Syndrome (Non STE ACS) (comprising of Non ST elevation myocardial infarction and unstable angina) was the indication in majority of the study population 405(46.1\%) followed by STEMI in 290(32.9\%) and positive stress test in $184(21 \%)$ patients in both groups (Table 2). Isolated CAE (without stenotic CAD) was uncommon and was seen in only $32(15.5 \%)$ patients. The remaining $175(84.5 \%)$ patients had ectasia and stenosis in the same or other vessels. There were 29(90.6\%) males and $3(9.4 \%)$ females in isolated CAE while $166(94.6 \%)$ males and $9(5.1 \%)$ females in CAE with stenosis. Hypertension was present in 18(56.3\%) isolated CAE and 99(56.6\%) CAE with stenosis. Diabetes mellitus was present in $9(28.1 \%)$ isolated CAE and 67(38.3\%) CAE with coronary stenosis. Combined Hypertension and DM was present in $8(25 \%)$ isolated CAE and 50(28.6\%) CAE with coronary stenosis. Dyslipidemia was present in $12(37.5 \%)$ isolated CAE and $49(28 \%)$ CAE with stenosis. Smoking was present in $16(50 \%)$ isolated CAE and $94(53.7 \%)$ CAE with stenosis.

Table 2 Indications for coronary angiopraphy

\begin{tabular}{llll}
\hline Characteristics & $\begin{array}{l}\text { CAE Group } \\
\mathbf{N = 2 0 7}\end{array}$ & $\begin{array}{l}\text { Stenotic Cad } \\
\text { Group N=672 }\end{array}$ & p value \\
\hline Non STE ACS & $96(46.1 \%)$ & $309(45.9 \%)$ & $<0.677$ \\
STE ACS & $66(32.9 \%)$ & $224(33.3 \%)$ & $<0.097$ \\
Positive stress test & $45(21 \%)$ & $139(20.7 \%)$ & $<0.819$ \\
\hline
\end{tabular}

STE ACS: ST Segment Elevation Acute Coronary Syndromes.

In CAE group, single vessel CAE was observed in 124 patients $(59.9 \%)$ patients, two vessel CAE was seen in 44 patients $(21.3 \%)$ and three vessel CAE was found in only 39 patients (18.8\%). In older 
patients ( $>60$ years old) one vessel ectasia was seen in $46(63.9 \%)$ while in $<60$ years old patients it was present in $78(57.8 \%)$. In $>60$ years old two vessel ectasia was observed in $14(19.4 \%)$ and in $<60$ years old patients it was seen in $30(22.2 \%)$. Three vessel ectasia was seen in $12(16.7 \%)$ patients in $>60$ years of age and it was observed in $27(20 \%)$ in $<60$ years old.

The distribution of stenosis in various coronary arteries was compared in CAE and Stenotic CAD Groups (Table 3). Overall, coronary stenosis in LAD was observed in $477(70.9 \%)$ in Stenotic CAD Group compared to $117(56.5 \%)$ patients in CAE Group $(\mathrm{p}<0.0001)$. LCx stenosis was more frequent in Stenotic CAD Group of $342(50.8 \%)$ vs. $54(26.1 \%)$ in CAE Group ( $<0.0001)$. In RCA, stenosis was observed in 507(75.3\%) in Stenotic CAD Group vs. 94(45.4\%) to CAE Group ( $<<0.0001)$ (Table 3).

Table 3 Presence of significant coronary artery stenosis in Ectasia vs Nonectasia groups

\begin{tabular}{llll}
\hline Characteristics & $\begin{array}{l}\text { CAE Group } \\
\mathbf{N = 2 0 7}\end{array}$ & $\begin{array}{l}\text { Stenotic Cad } \\
\text { Group N=672 }\end{array}$ & p value \\
\hline RCA & $94(45.4 \%)$ & $507(75.3 \%)$ & $<0.000$ I \\
LAD & $117(56.5 \%)$ & $477(70.9 \%)$ & $<0.0001$ \\
LCX & $54(26.1 \%)$ & $342(50.8 \%)$ & $<0.0001$ \\
LMS & $7(3.4 \%)$ & $42(6.2 \%)$ & $<0.117$ \\
\hline
\end{tabular}

LAD: Left Anterior Descending; LCX: Left Circumflex; LMS: Left Main Stem; RCA: Right Coronary Artery

In order to study the association of CAE with CAD, we studied the presence of stenotic $\mathrm{CAD}$ in the vessels having ectasia versus Non ectatic vessels of the same patients. Left main stem ectasia was observed in 15(7.3\%) patients of whom 14(93.3\%) also had stenoses in other coronary arteries, only one $(6.7 \%)$ having both stenosis and CAE in left main. LAD ectasia was found in $82(39.6 \%)$ patients, $65(79.3 \%)$ had stenoses in other coronary arteries while $33(40.2 \%)$ had simultaneous presence of CAD with CAE in the LAD itself. LCx ectasia was found in 73(35.3\%) patients, 61(83.6\%) had CAD in other coronary arteries while $14(19.2 \%)$ had simultaneous presence of CAD with ectasia in the LCx itself. RCA ectasia was observed in $126(60.7 \%)$ patients, $100(79.4 \%)$ had CAD in other coronary arteries while $47(37.3 \%)$ had simultaneous presence of CAD with ectasia in the RCA itself.

\section{Discussion}

As compared to patients with stenotic coronary artery disease the current study reveals that patient with coronary ectasia were younger and are less likely to be diabetic. Of the four age segments, CAE was more common in the younger age group, while stenotic CAD was common in older age. This is in agreement with previous studies. ${ }^{10-13}$ Giannoglu et al., ${ }^{11}$ also reported more male patients having CAE. This gender difference has been reported previously ${ }^{12,13}$ and is supposed to be due to a lower incidence of CAD in women. ${ }^{11}$ Considering the different age segments, we found nearly three quarters of CAE to be younger than 60years. This is different from other regions not only CAE occurs at younger age in our region in the INTERHEART study, Gehani et al., ${ }^{14}$ reported that the age at first AMI was $51.2 \pm 10.3$ years, which was the youngest in the entire INTERHEART population. ${ }^{14}$ ,who reported that majority of patients with CAE were males in their sixth decade. ${ }^{15}$ Age has been shown to constitute a significant factor that is inversely related to the presence of CAE. ${ }^{11}$ The higher likelihood of males having CAE compared with women is generally consistent with a study in Spain in which male gender was demonstrated to be an independent factor that increased the hazard of CAE. ${ }^{16}$
Diabetes mellitus is a well known risk factor for coronary atherosclerosis and its complications. ${ }^{17-19}$ Several recent studies have evaluated and compared traditional cardiovascular risk factors in patients with CAE and in those with stenotic CAD. As in our study, an interesting observation is the negative correlation between diabetes and CAE (36.7\% in CAE vs $48.3 \%$ in CAD) has also been reported by others. The prevalence of diabetes in patients with ectasia was found to be less frequent than that in patients with stenotic CAD. ${ }^{3,16}$

Androulakis et al., ${ }^{3}$ and Bermudez et al., ${ }^{16}$ reported significant, independent and inverse association between CAE and diabetes mellitus. Diabetes mellitus is known to promote negative remodeling in the arterial wall and impairs compensatory arterial enlargement during the course of the atherosclerotic process..$^{20}$ Therefore it may be reasonable to expect such an inverse association between diabetes mellitus and CAE. This inverse relationship also suggests that the pathogenesis underlying CAE may not simply be a variant of coronary atherosclerosis.

Sayin et al., ${ }^{21}$ postulated that CAE renders patients at higher risk of myocardial ischemia irrespective of extent of stenosis. ${ }^{21}$ Nayamu et al.,22 reported a significant proportion of patients at presentation either had an acute myocardial infarction or a history of myocardial infarction while $(49 \%)$ patients had angina. Similar to these findings Demopoulos et al., ${ }^{10}$ reported $39 \%$ of patients to have angina or myocardial infarction. ${ }^{10} \mathrm{We}$ observed similar proportion of patients in CAE and Stenotic CAD group to have Non STEMI, unstable angina and STEMI. Patients with CAE without stenosis had significantly more frequent abnormal treadmill stress tests compared with those with normal coronary angiography, suggesting decrease in coronary flow reserve and microvascular dysfunction as a possible contributing factor for myocardial ischemia in CAE. Hartnell ${ }^{12}$ and Markis ${ }^{23}$ reported that angina is the most common presenting complaint in patients with CAE. However in our study ACS is the most common presentation in patients with CAE.

We found the distribution of CAE and stenotic CAD to be different among major coronary arteries. CAE was most frequently observed in LAD followed by RCA and LCx. Conversely stenotic CAD was most frequent in RCA followed by LAD and LCX.

CAE has a high coexistence with CAD in our study. Approximately $85 \%$ of patients with CAE also had concomitant stenosing CAD. This finding is consistent with other studies. ${ }^{10,23,24}$ In these patients with concomitant disease, the right coronary artery is most frequently involved in the ectatic process, followed by the left anterior descending artery and the circumflex artery. ${ }^{10,24}$

Some previous studies also found that the RCA is the most commonly involved coronary artery in patients with isolated CAE ranging from $45 \%$ to $75 \%$ (this was $45.4 \%$ in our study). ${ }^{10,23}$ In our study RCA stenosis was highest $75.3 \%$ followed by LAD stenosis $70.9 \%$ and LCx stenosis in 50.8\%. In Demopoulos's report involvement of the RCA by the stenotic lesion was the least compared to LAD and LCx. ${ }^{10}$ Demopoulos's reported that in patients with CAD coexisting with CAE, $34 \%$ of the stenotic lesions were in the vessels affected by the ectatic process, while $65 \%$ were in the non-ectatic vessels. ${ }^{10}$

In current study, Left main stem ectasia was observed in 15 patients of whom $14(93.3 \%)$ also had stenoses in other coronary arteries, only one $(6.7 \%)$ having both stenosis and CAE in left main. Previous studies have reported all three coronary vessels to be affected by CAE, but in almost $75 \%$ of patients an isolated artery was found to be ecstatic. ${ }^{25,26}$ The reason for the higher RCA predisposition to CAE is not well understood. In a small percentage of patients, CAE does not coexist with coronary stenosis. ${ }^{8}$ 


\section{Conclusion}

Compared to CAD, coronary ectasia occurred in younger male patients and inversely related to diabetes. ACS is the most common presentation for patients with CAE which suggest that it is not just a benign condition. The distribution of CAE among the three coronary arteries is also different to that in CAD.

\section{Acknowledgments}

None.

\section{Conflicts of interest}

Author declares there are no conflicts of interest.

\section{Funding}

None.

\section{References}

1. Go AS, Mozaffarian D, Roger VL, et al. On behalf of the American Heart Association Statistics Committee and Stroke Statistics Subcommittee. Heart Disease and Stroke Statistics-2013 Update. A Report From the American Heart Association. Circulation. 2013;127(1):e6-e245.

2. Boles U, Eriksson P, Zhao Y, et al. Coronary artery ectasia: remains a clinical dilemma. Coron Artery Dis. 2010;21(5):318-320.

3. Androulakis AE, Andrikopoulos GK, Kartalis AN, et al. Relation of coronary artery ectasia to diabetes mellitus. Am J Cardiol. 2004;93(9):1165-1167.

4. Aboeata AS, Sontineni SP, Alla VM, et al. Coronary artery ectasia:current concepts and interventions. Front Biosci (Elite Ed). 2012;4:300-310.

5. Valente S, Lazzeri C, Giglioli C, et al. Clinical expression of coronary artery ectasia. J Cardiovasc Med (Hagerstown). 2007;8(10):815-820.

6. Manginas A, Cokkinos DV. Coronary artery ectasias: imaging, functional assessment and clinical implications. Eur Heart $J$. 2016;27(9): 1026-1031.

7. Saglam M, Karakaya O, Barutcu I, et al. Identifying cardiovascular risk factors in a patient population with coronary artery ectasia. Angiology. 2007;58(6):698-703.

8. Mavrogeni S. Coronary artery ectasia from diagnosis to treatment Hellenic J Cardiol . 2010;51:158-163.

9. Demopoulos VP, Olympios CD, Fakiolas CN, et al. The natural history of aneurysmal coronary artery disease. Heart. 1997;78(2):136-141

10. Leschka S, Stolzmann P, Scheffel H, et al. Prevalence and morphology of coronary artery ectasia with dual-source CT coronary angiography. Eur Radiol. 1997;18(12):2776-2784.

11. Giannoglou GD, Antoniadis AP, Chatzizisis YS, et al. Prevalence of Ectasia in Human Coronary Arteries in Patients in Northern Greece Referred for Coronary Angiography. Am J Cardiol. 2006;98(3):314-318.
12. Hartnell GG, Parnell BM, Pridie RB. Coronary artery ectasia: its prevalence and clinical significance in 4993 patients. Br Heart $J$. 1985;54(4):392-395.

13. Swanton RH, Thomas ML, Coltart DJ, et al. Coronary artery ectasia: a variant of occlusive coronary arteriosclerosis. $\mathrm{Br}$ Heart $J$. 1978;40(4):393-400.

14. Gehani AA, Al-Hinai AT, Zubaid M, et al. Association of risk factors with acute myocardial infarction in Middle Eastern countries: the INTERHEART Middle East study. Eur $J$ Prev Cardiol. 2014;21(4):400-410.

15. Lam CS, Ho KT. Coronary Artery Ectasia: A Ten years experience in a Tertiary Hospital in Singapore. Ann Acad Med Singapore. 2004;33(4):419-422.

16. Pinar Bermúdez E, López Palop R, Lozano Martínez-Luengas I, et al. Coronary ectasia: prevalence, and clinical and angiographic characteristics. Rev Esp Cardiol. 2003; 56(5):473-479.

17. Assmann G, Cullen P, Schulte H. Simple scoring scheme for calculating the risk of acute coronary events based on the 10-year follow-up of the Prospective Cardiovascular Münster (PROCAM) study. Circulation. 2002;105(3):310-315.

18. Fujiwara S, Emoto M, Komatsu M, et al. Arterial wall thickness is associated with insulin resistance in type 2 diabetic patients. $J$ Atheroscler Thromb. 2003;10(4):246-252.

19. Kannel WB, McGee DL. Diabetes and cardiovascular disease: the Framingham study. JAMA . 1979;241(9):2035-2038.

20. Virmani R, Robinowitz M, Atkinson JB, et al. Acquired coronary arterial aneurysms: an autopsy study of 52 patients. Hum Pathol. 1986;17(6):575-583.

21. Sayin T, Döven O, Berkalp B, et al. Exercise-induced myocardial ischemia in patients with coronary artery ectasia without obstructive coronary artery disease. Int J Cardiol. 2001;78(2):143-149.

22. Sultana R, Sultana N, Ishaq M, et al. The Prevalence and Clinical Profile of Angiographic Coronary Ectasia. J Pak Med Assoc. 2011;61(4):372-375.

23. Markis JE, Joffe CD, Cohn PF, et al. Clinical significance of coronary arterial ectasia. Am J Cardiol. 1976;37(2):217-222.

24. Farto-e-Abreu P, Mesquita A, Silva JA, et al. Coronary artery ectasia: clinical and angiographic characteristics and prognosis. Rev Port Cardiol. 1993;12(4):305-310.

25. Daoud AS, Pankin D, Tulgan H, et al. Aneurysms of the coronary artery. Report of ten cases and review of literature. Am J Cardiol. 1963;11:228-237.

26. Al-Harthi SS, Nouh MS, Arafa M, et al. Aneurysmal dilatation of the coronary arteries: diagnostic patterns and clinical significance. Int $J$ Cardiol. 1991;30(2):191-194. 